\title{
The correlation of microRNA-181a and target genes with poor prognosis of glioblastoma patients
}

\author{
SHI-XIONG HUANG ${ }^{1}$, ZHONG-YAN ZHAO ${ }^{1}$, GUO-HU WENG ${ }^{1}$, XIANG-YING HE ${ }^{1}$, \\ CHAN-JI WU ${ }^{1}$, CHUAN-YI FU ${ }^{2}$, ZHI-YAN SUI ${ }^{1}$, XIAO-MING ZHONG ${ }^{3}$ and TAO LIU $^{1}$ \\ Departments of ${ }^{1}$ Neurology and ${ }^{2}$ Neurosurgery, People's Hospital of Hainan Province, Haikou, Hainan 570311; \\ ${ }^{3}$ Department of Radiology, Ganzhou City People's Hospital, Ganzhou, Jiangxi 341000, P.R. China
}

Received February 2, 2016; Accepted March 15, 2016

DOI: $10.3892 /$ ijo.2016.3511

\begin{abstract}
To investigate the expression and clinical significance of miR-181a and its target genes in glioblastoma multiforme (GBM), the expression levels of miR-181a and three target genes in human normal brain tissues and GBM were analyzed in silico using gene microarray, Gene Ontology, KEGG pathway and hierarchical clustering analysis followed by validation with quantitative RT-PCR. Our results show that miR-181a is down-regulated in GBM patients. The three target genes, ANGPT2, ARHGAP18 and LAMC1, are negatively correlated with the expression of miR-181a. Moreover, high expression of ANGPT2 or LAMC1 together with large size of GBM is correlated with a shorter median overall survival. In conclusion, our results showed that miR-181a and it targets ANGPT2 and LAMC1 might be predictors of prognosis in GBM patients.
\end{abstract}

\section{Introduction}

Glioma is the most common malignant tumor in the central nervous system, with incidence rate of 5-7 per 100,000 and 5 -year survival rate of approximately $20-30 \%$ (1). Gliomas originate from astrocytes, oligodendrocytes and neural stem cells or their precursors. According to WHO classification, gliomas are classified into four different malignant grades ranging from grade I to grade IV based on histopathological features and related molecular aberrations, of which the glioblastoma multiforme (GBM) is classified as grade IV, the most aggressive group and one of the hardest tumors to treat (2). The therapy method of GBM mainly include surgery, radiation and chemotherapy, but the survival of patients is still quite short, especially when invasion and metastasis occur (3).

Correspondence to: Dr Tao Liu, Department of Neurology, People's Hospital of Hainan Province, Haikou, Hainan 570311, P.R. China

E-mail:1tao829@126.com

Key words: glioblastoma multiforme, miR-181a, target genes, GO analysis, overall survival, prognosis
To improve the prognosis of GBM, great efforts have been taken to identify molecular markers and therapeutic targets. In which, several genes, such as RINT1, mTORC2 and WT1 have been reported to participate in the carcinogenesis and progression of GBM (4). However, there is no biomarker with enough sensitivity and specificity for assessment of response to therapy and prognosis in GBM. Thus, a better understanding of the mechanisms involved in regulating tumor growth requires the identification of novel genes is associated with GBM.

MicroRNAs (miRNAs) are small 19-24 nucleotide, noncoding RNAs that can inhibit the post-transcription of target mRNA by completely or incompletely matching with the target mRNA, and then participate in the regulation of many biological processes, such as cell proliferation, metabolism, differentiation and apoptosis (5). Therefore, aberrant miRNA expression associated with the development of different kinds of cancer.

Accumulated evidence indicates that several mRNAs and genes, respectively participate in the progression of GBM. Currently, global analyses have revealed that several miRNAs are clinically implicated with GBM, such as miR103a, miR-107 and miR-182 (6). Previous reports showed that miR-372 regulates GBM cell proliferation and invasion by directly targeting PHLPP2 (7). miR-210 upregulation inhibits proliferation and induces apoptosis in GBM cells by targeting SIN3A (8) and miR-429 inhibits GBM invasion through BMK1 suppression (9).

However, no previous report exists on investigation of the correlation between the expression of miR-181a and target genes in GBM. We profiled miRNAs and genes expression by microarray to identify their differentially expression in GBM and normal brain tissues, and then explored the correlation between miR-181a and its target genes in GBM to provide further insight into the pathogenesis of GBM.

\section{Materials and methods}

Ethics statement. The study protocol and acquisition of tissue specimens were approved by the Ethics Committee of the Ganzhou City People's Hospital (2015-RES-10). Each participant provided written informed consent before participating in the present study. 
Acquisition of clinical specimens. GBM samples were collected from patients undergoing surgical resection and classified according to the last WHO classification of central nervous tumors and clinical histories were recorded, confirmed by two experienced pathologists independently.

Collection of clinical information and follow up. Demographic and clinicopathological characteristics were recorded including the patient's characteristics (e.g., age and gender), tumor characteristics (size, necrosis, boundary and cystic degeneration), overall survival time. All previous data were collected by reviewing the clinical history. For analysis, patients were stratified according to age, $\geq 40$ or $<40$ years. The tumor size was described by mean tumor diameter (MTD, defined as the geometric mean of 3 diameters on MRI scan), and tumors were grouped according to size, $\geq 5 \mathrm{~cm}$ and $<5 \mathrm{~cm}$. The followup was conducted by telephone or direct correspondence. The time of tumor relapse or death was verified by the patient or their relatives, by medical recording, or by the social security record. Overall survival (OS) was calculated in months from the date of diagnosis to the time of death, regardless of cause.

RNA extraction. According to the manufacturer's guideline, total RNA was isolated using TRIzol reagent (Invitrogen, Carlsbad, CA, USA). RNA quantity was determined using NanoDrop ND-1000 spectrophotometer and the integrity of RNA was measured by gel electrophoresis.

Gene microarray analysis. For gene expression microarray analysis, tumor tissue from 22 GBM patients was assessed on an Affymetrix array platform (Affymetrix, Inc., Santa Clara, CA, USA). The sample preparation and microarray hybridization were performed based on the manufacturer's standard protocols and published studies (10-13).

Cell lines and transfection. Human glioblastoma cell line U251 were purchased from Shanghai Institute for Biological Sciences (Shanghai, China) and cultured in Dulbecco's modified Eagle's medium (DMEM; Gibco, Carlsbad, CA, USA) supplemented with $10 \%$ fetal bovine serum (FBS; HyClone Laboratories, Inc., Logan, UT, USA), 100 units/ml penicillin and $100 \mathrm{mg} / \mathrm{ml}$ streptomycin at $37^{\circ} \mathrm{C}$ in a humidified chamber supplemented with $5 \% \mathrm{CO}_{2}$.

miR-181a mimic and corresponding negative control miRNA (miR-NC) were purchased from Shanghai GenePharma, Co., Ltd. (Shanghai, China). These molecular productions were transiently transfected into U251 cells, respectively, using Oligofectamine ${ }^{\mathrm{TM}}$ transfection reagent (Invitrogen) according to the manufacturer's instructions.

Reporter assays. The human ANGPT2, ARHGAP18 or LAMC1 3'-UTR oligonucleotides containing the wild-type (Wt) or mutant (Mut) miR-181a binding site were subcloned into the XhoI and Not I sites of the psiCHECK2 vector (Promega, Madison, WI, USA). For luciferase assay, U251 cells were inoculated into 24-well plates and cultured for $24 \mathrm{~h}$; then, cells were co-transfected with the Wt/Mut reporter plasmid (100 ng) and miR-181a mimic/miR-NC (100 nM). Forty-eight hours after the transfection, luciferase assay was determined using the Dual-Luciferase kit (Promega).
Quantitative RT-PCR ( $q R T-P C R)$. For qRT-PCR of ANGPT2, ARHGAP18 and LAMC1, cDNA was synthesized from total RNA (10 ng), and quantitative PCR reactions were performed with the TaqMan ${ }^{\text {TM }}$ Universal PCR kit (Life Technologies, Grand Island, NY, USA). GAPDH was used as the internal control. Quantitative qRT-PCR for miR-181a was performed using the TaqMan Universal PCR kit (Life Technologies). U6 small nuclear RNA was used as internal control, and the $2^{-\Delta \Delta C T}$ method was used to analyze the expression levels of miR-181a and 3 gene levels.

Bioinformatic analysis. The expression levels of miRNAs were investigated in GBM and normal tissue samples in the GEO datasets using the NCBI Platform (http://www.ncbi. nlm.nih.gov/). Hierarchical clustering was performed using the multiple experiment viewer (MeV) 4.7.1 software (http:// www.tm4.org/mev/). Each row represents a miRNA or gene, and each column represents a sample in the heat map diagram. The clustering tree of miRNAs or genes are shown on the left, and the sample clustering tree appears at the top.

We used the target gene prediction software DIANATarBase (14), TargetScan (http://www.targetscan.org) and PicTar (http://pictar.mdc-berlin.de/) to forecast several potential target genes of miR-181a.

To elucidate the relationship between gene differential expression patterns, we used GO classifications (http://www. geneontology.org/), which mainly analyzed three aspects including biological process, molecular function and cellular components, which reflect the biological function of differential expression genes in GBM. Subsequent bioinformatic analysis of these genes was performed by the Kyoto Encyclopedia of Genes and Genomes Pathway analysis (KEGG Pathway analysis) (http://www.genome.jp/kegg/). Pathways were selected with a $\mathrm{P}$-value $<0.05$ and gene count $>2$.

Statistical analysis. The results were expressed as mean \pm SD (standard deviation). The statistical significance between the groups was assessed by using one-way analysis of variance (ANOVA). Univariate survival analysis and multivariate analyses were carried out using the Kaplan-Meier method. All calculations were performed with the SPSS 20.0 software program (SPSS, Inc., Chicago, IL, USA). The level of significance was chosen as $\mathrm{P}<0.05$.

\section{Results}

miRNAs microarray analysis. The expression profiles of human miRNAs were analyzed by microarrays in three paired GBM tissues download from the Gene Expression Omnibus (GEO) database (GSE65626-GPL19117). After hierarchical clustering of miRNAs, the GBM samples and normal samples were completely separated. Of all the miRNAs represented on the array, 39 miRNAs displayed at least a 2-fold increased or 0.5 -fold decreased in expression at the $\mathrm{P}<0.01$ level with a false discovery rate. Of which, 30 miRNAs were upregulated in human GBM tissues, while 9 miRNAs were downregulated in human GBM tissues (Fig. 1A). Several common miRNAs, such as miR-21, miR-10b and miR-27a, linked to the risk of GBM. However, no previous report exists on investigation of the correlation between the expression of miR-181a and the 

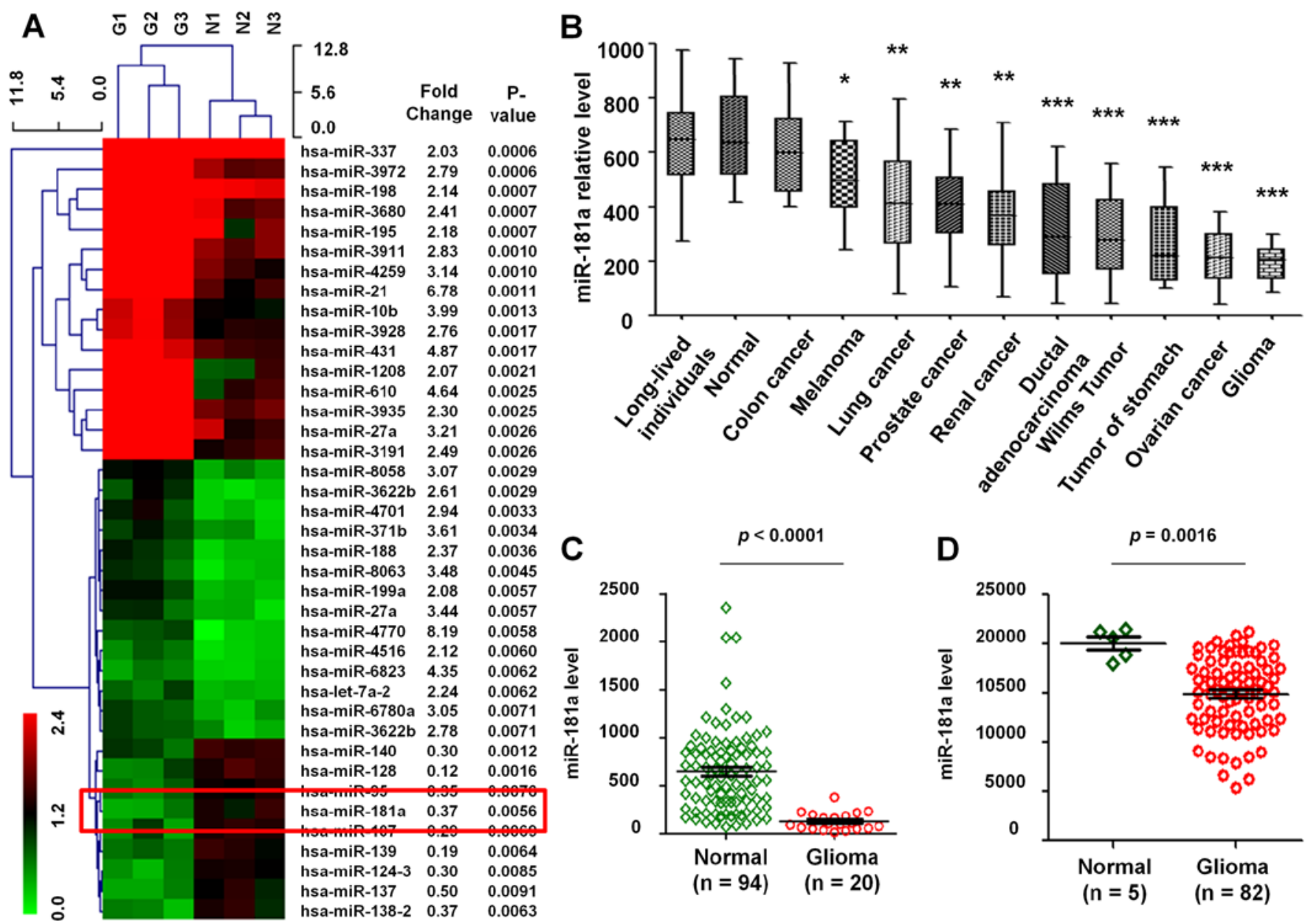

Figure 1. The expression of miR-181a in normal and GBM tissues. (A) Hierarchical clustering for differential miRNAs in 3 paired GBM tissues derived from GEO database (GSE65626-GPL19117). (B) Expression levels of miR-181a in long-lived individuals, normal tissues and various cancers derived from the GEO database (GSE61741). Expression levels of miR-181a in GBM vs. normal tissues derived from the (C) GEO dataset GSE61741 and (D) GSE25632-GPL8179.

target genes in GBM. Moreover, we found miR-181a that is rarely referred to presented downregulation in GBM.

Subsequently, we analyzed the expression level of the above mentioned differential miRNAs in 94 normal controls and 448 various cancers included 20 GBM from the GEO datasets (GSE61741). This project analyzed peripheral blood miRNA profiles and each miRNA was measured in at least seven replicates, the median of the replica had been computed. The results showed that the expression level of miR-181a in various tumors was lower than in normal samples (Fig. 1B). Then, 94 normal and 20 GBM samples were used to evaluate the expression profile of miR-181a. Results show that the expression level of miR-181a in GBM was remarkably lower than in normal tissues $(\mathrm{P}<0.0001$, fold change, 0.21; Fig. 1C).

In addition, data of 5 normal and 82 GBM samples from the GEO database (GSE25632-GPL8179) were also analyzed, which similarly reflected that miR-181a was downregulated in GBM compared to normal tissues ( $\mathrm{P}=0.0016$, fold change, 0.66; Fig. 1D), thus miR-181a was downregulated in tumors especially in GBM.

Gene microarray analysis. We further analyzed gene expression profile of the above three paired GBM tissue samples. Of all the genes represented, 46 genes displayed at least a 1.5-fold increased or 0.5 -fold decreased in expression at the $\mathrm{P}<0.01$ level. Of which, 17 genes represent upregulated function, while 29 genes were downregulated in human GBM tissues (Fig. 2A).

GO and KEGG pathway analysis. In biological process, the top 5 GO terms of these 46 differential genes are neuroblast proliferation, protein folding, blood vessel development, tube development and transcription. In molecular function, the top 5 GO terms are transcription regulator activity, transcription repressor activity, transcription cofactor activity, transcription factor binding and tubulin binding. The top $5 \mathrm{GO}$ terms in cellular component are intracellular organelle lumen, organelle lumen, membrane-enclosed lumen, nuclear lumen and nucleoplasm (Fig. 2B).

KEGG pathways analysis of these differentially expressed gene patterns revealed several enrichment-related pathways, including pathways in apoptosis, ECM-receptor interaction, pathways in cancer, histidine metabolism and focal adhesion (Fig. 2B).

TCGA dataset comparative analysis. We obtained a total of 557 GMB cases with level 3 gene expression data based 
A

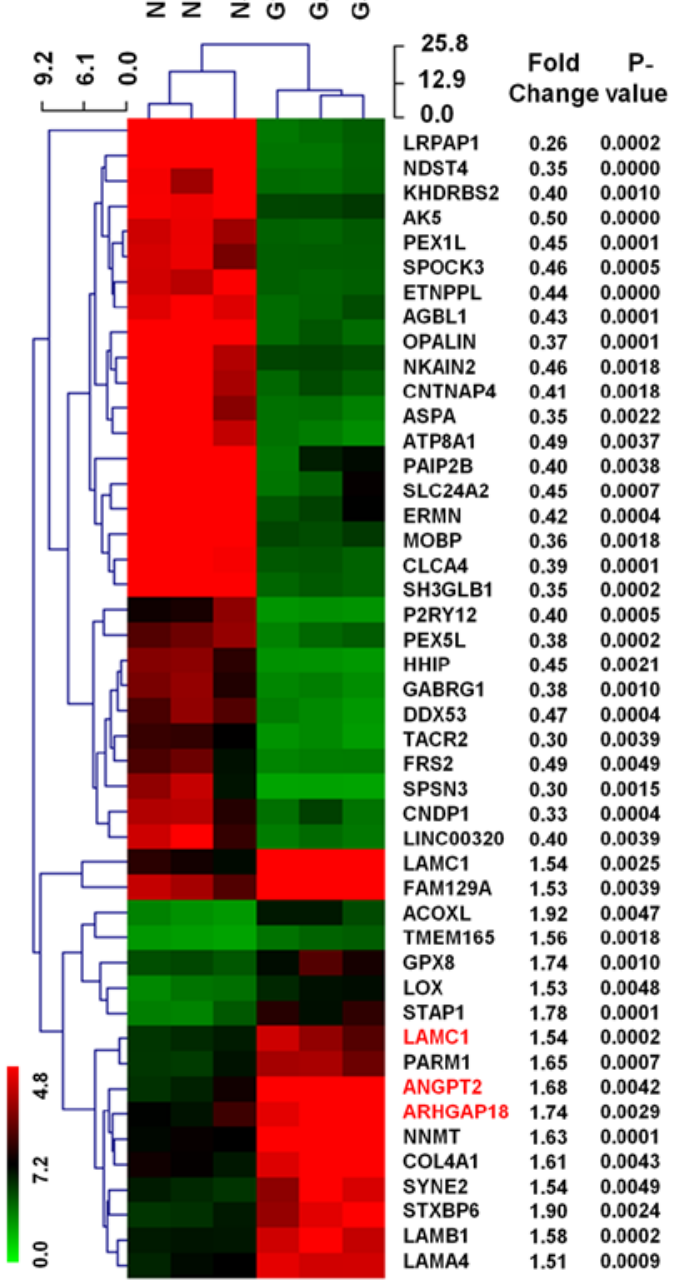

B

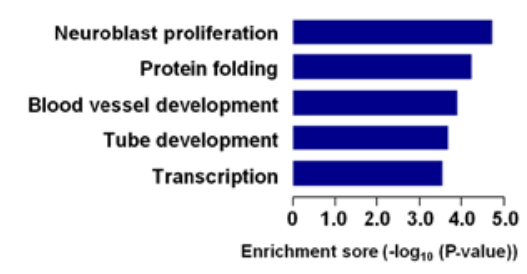

Cellular component

Intracellular organelle lumen

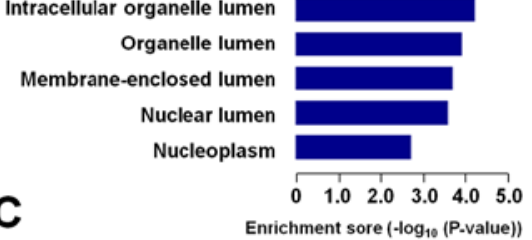

Molecular function

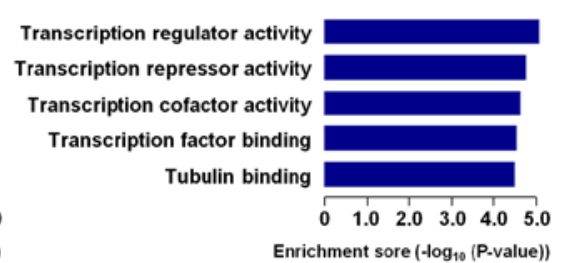

Enrichment sore (- $\log _{10}($ P-value))

KEGG pathway

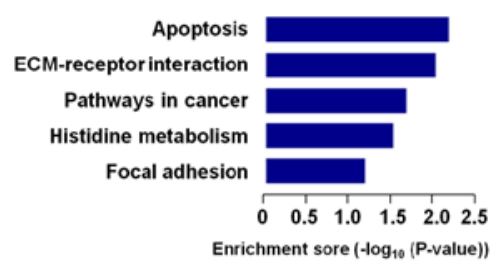

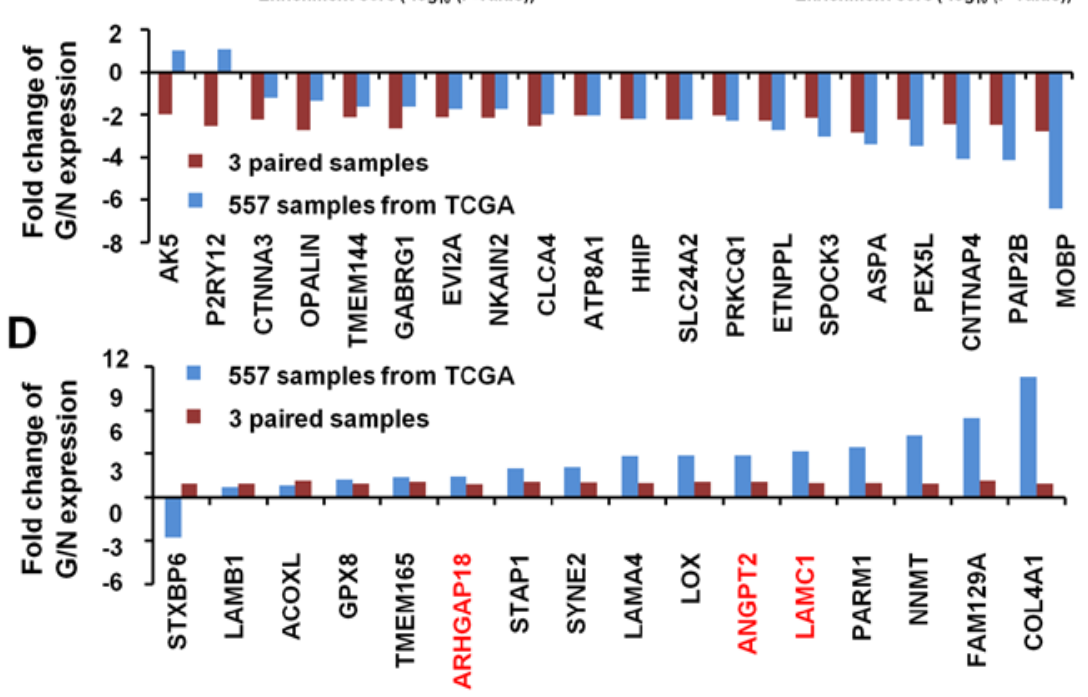

Figure 2. The expression of differential genes in normal and GBM tissues. (A) Hierarchical clustering of differential genes in 3 paired GBM tissues derived from GEO database (GSE65626-GPL19117). (B) GO analysis and KEGG pathway analysis of differential genes. (C) Downregulated and (D) upregulated genes expression in 3 paired GBM vs. 557 glioma samples from the TCGA database.

on microarray by search of the TCGA dataset. In contrast to standard TCGA dataset on gene expression, we found that upregulated genes (in our 3 paired samples) are largely in keeping with TCGA dataset in fold change of GBM (Fig. 2C). In addition, downregulated genes are also mostly in accordance with TCGA dataset in fold change of expression in GBM (Fig. 2D).

Computational prediction of associations between miRNAs and target genes. To elucidate whether there were paired miRNAs and target mRNAs profiles aberrantly expressed in GBM, we used target gene prediction software including DIANA-TarBase, TargetScan and PicTar to search several potential target genes of miR-181a. There were 1750, 5019 and 427 predicted target genes found correlated with miR-181a in DIANA-TarBase, TargetScan and PicTar, respectively (Fig. 3A). Based on the analysis of the three databases, we found 111 common target genes.

Then, we analyzed the expression of these common target genes in 3 paired GBM and normal tissues mentioned before and found the negative relationships between the expression level of miR-181a and the predicted target genes (ANGPT2,
ARHGAP18 and LAMC1) either in normal brain (Fig. 3B) or in GBM tissues (Fig. 3C).

Besides, we analyzed the correlation between the expression level of miR-181a and 3 predicted target genes in 21 paired GBM samples from GEO dataset (GPL8179). As shown in Fig. 3D, the relationship between miR-181a expression level and ANGPT2 expression level presented inverse correlation $\left(\mathrm{R}^{2}=0.93\right)$. Similarly, the inverse correlation also emerged in miR-181a expression with mRNA levels of ARHGAP18 $\left(\mathrm{R}^{2}=0.91\right.$; Fig. 3E) and LAMC1 (R2=0.88; Fig. 3F).

Identification of ANGPT2, ARHGAP18 and LAMC1 as the miR-181a direct targets in U251 cells. To confirm the predicted target genes as direct targets of miR-181a, we performed luciferase reporter assays in U251 cells. As shown in Fig. 3G, transfection of miR-181a mimic caused a significant decrease in luciferase activity in cells transfected with the reporter plasmid with wild-type targeting sequence of mRNA, but not reporter plasmid with mutant sequence of the genes.

To confirm miR-181a regulation of the expression of ANGPT2, ARHGAP18 and LAMC1, we detected their expression in U251 cells after transfection with miR-181a or 

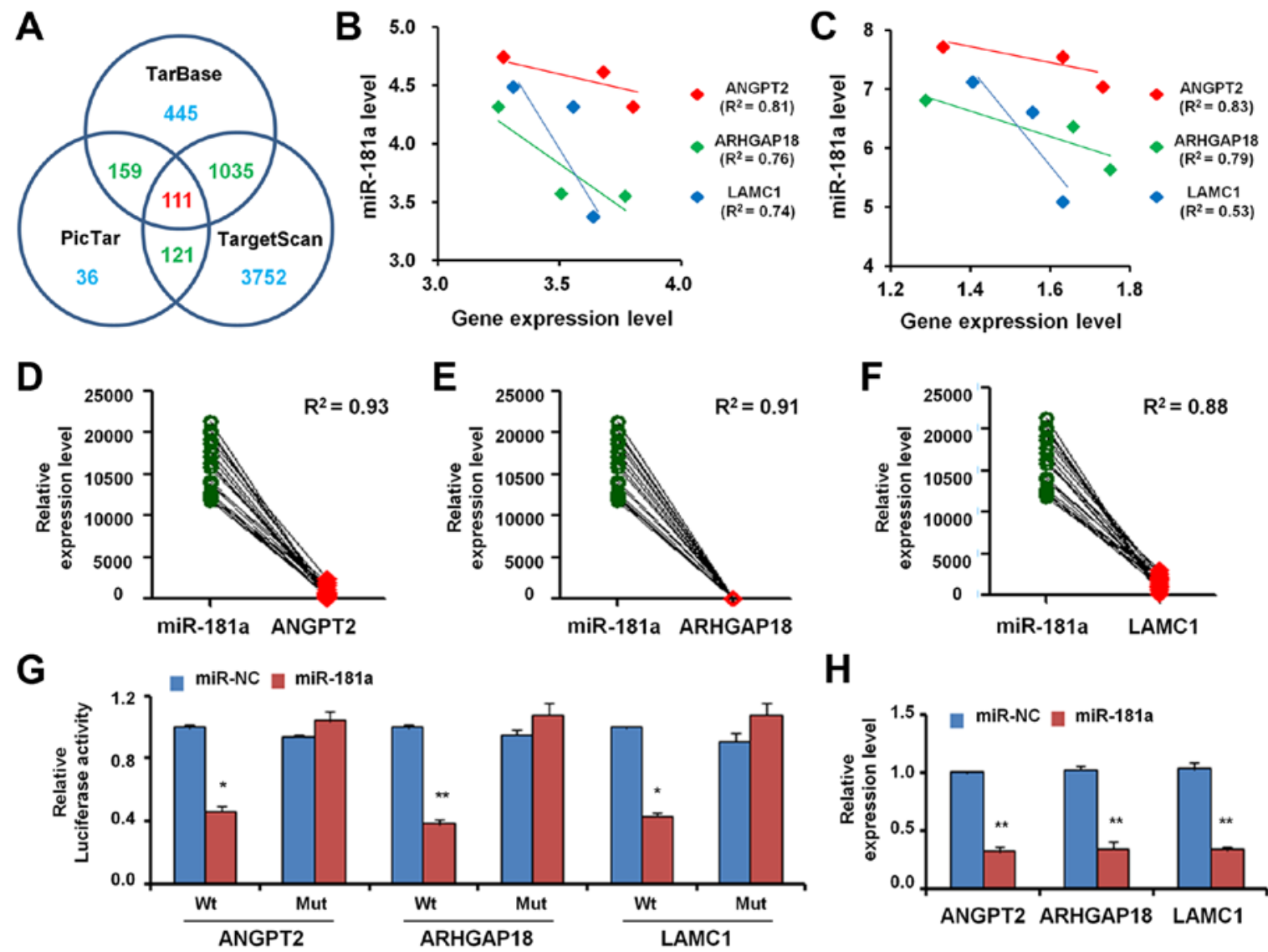

H
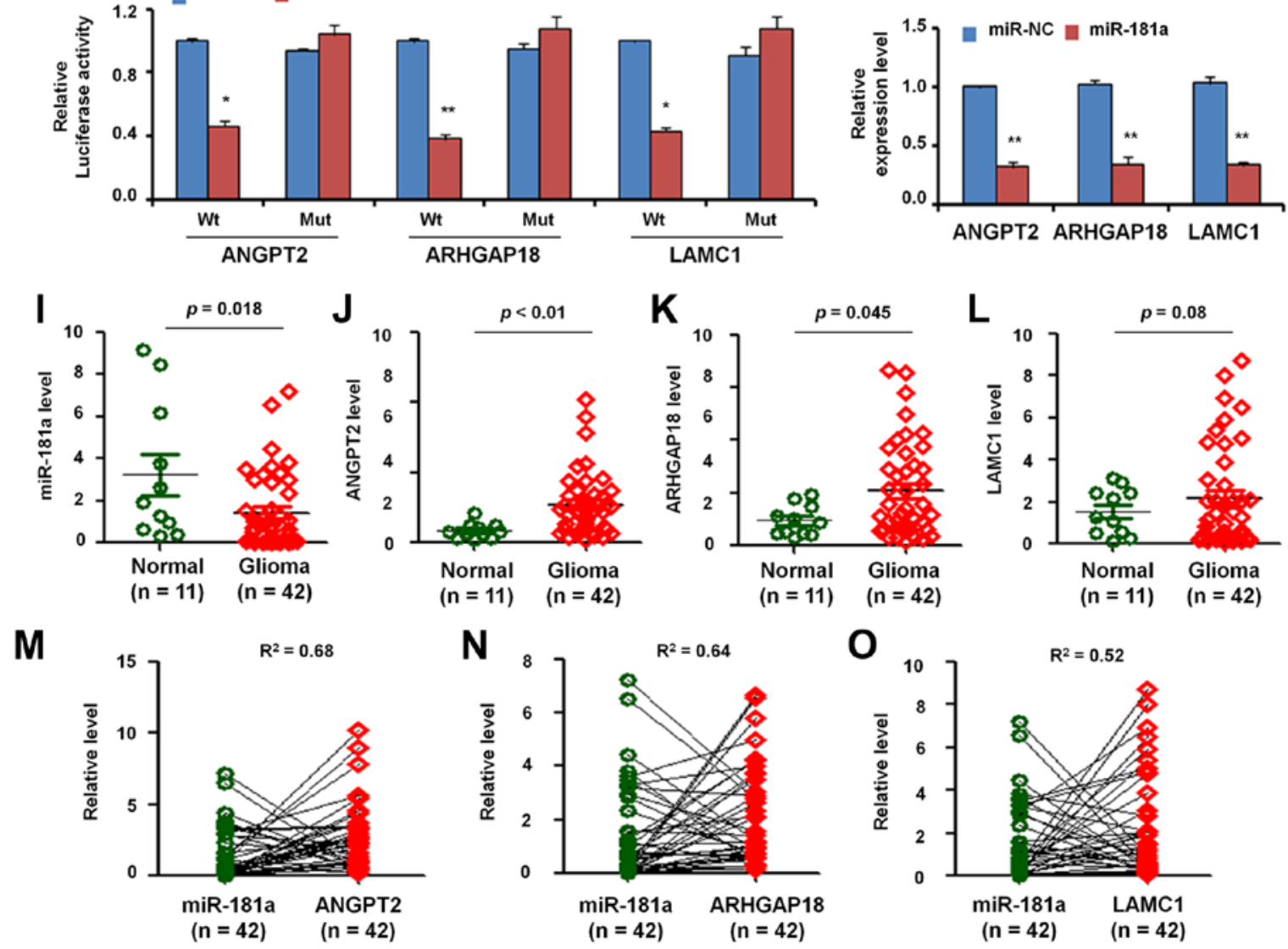

Figure 3. Relative expression of miR-181a and the target genes. (A) Result of target gene prediction for miR-181a by TarBase, TargetScan and PicTar software. The correlation of expression level of miR-181a with the predicted target genes in normal tissues (B) and GBM tissues (C) derived from the GEO database (GSE65626-GPL19117). Relative expression level of (D) miR-181a and ANGPT2, (E) ARHGAP18 or (F) LAMC1 in 3 GBM tissues derived from GEO database (GSE65626-GPL19117). (G) Analysis of luciferase activity in U251 cells co-transfected with miR-181a mimics or miR-NC and luciferase reporter plasmid (Wt/Mut 3'-UTR). (H) The expression of three endogenous genes on mRNA level were quantified in U251 cells after transfected with miR-181a mimic or miR-NC. The GAPDH was used for as an internal control. ${ }^{*} \mathrm{P}<0.05$; ${ }^{* *} \mathrm{P}<0.01$. (I) miR-181a, (J) ANGAT2, (K) ARHGAP18 and (L) LAMC1 expression level in 11 normal brain and 42 GBM tissues. Relative expression level of (M) miR-181a and ANGPT2, (N) ARHGAP18 or (O) LAMC1 in 42 GBM tissues.

miR-NC. It was found that miR-181a overexpression significantly inhibited mRNA expression level (Fig. 3H).

Validation of miR-181a/mRNAs expression and correlation using $q R T-P C R$. To further verify the correlation between miR-181a and the target genes, we extracted RNA from 11 normal brain tissues and 42 GBM tissues and carried out qRT-PCR. Fig. 3I shows that the expression level of miR-181a in GBM was lower than in normal tissues. However, ANGPT2 (Fig. 3J), ARHGAP18 (Fig. 3K) and LAMC1 (Fig. 3L) were overexpressed in GBM 

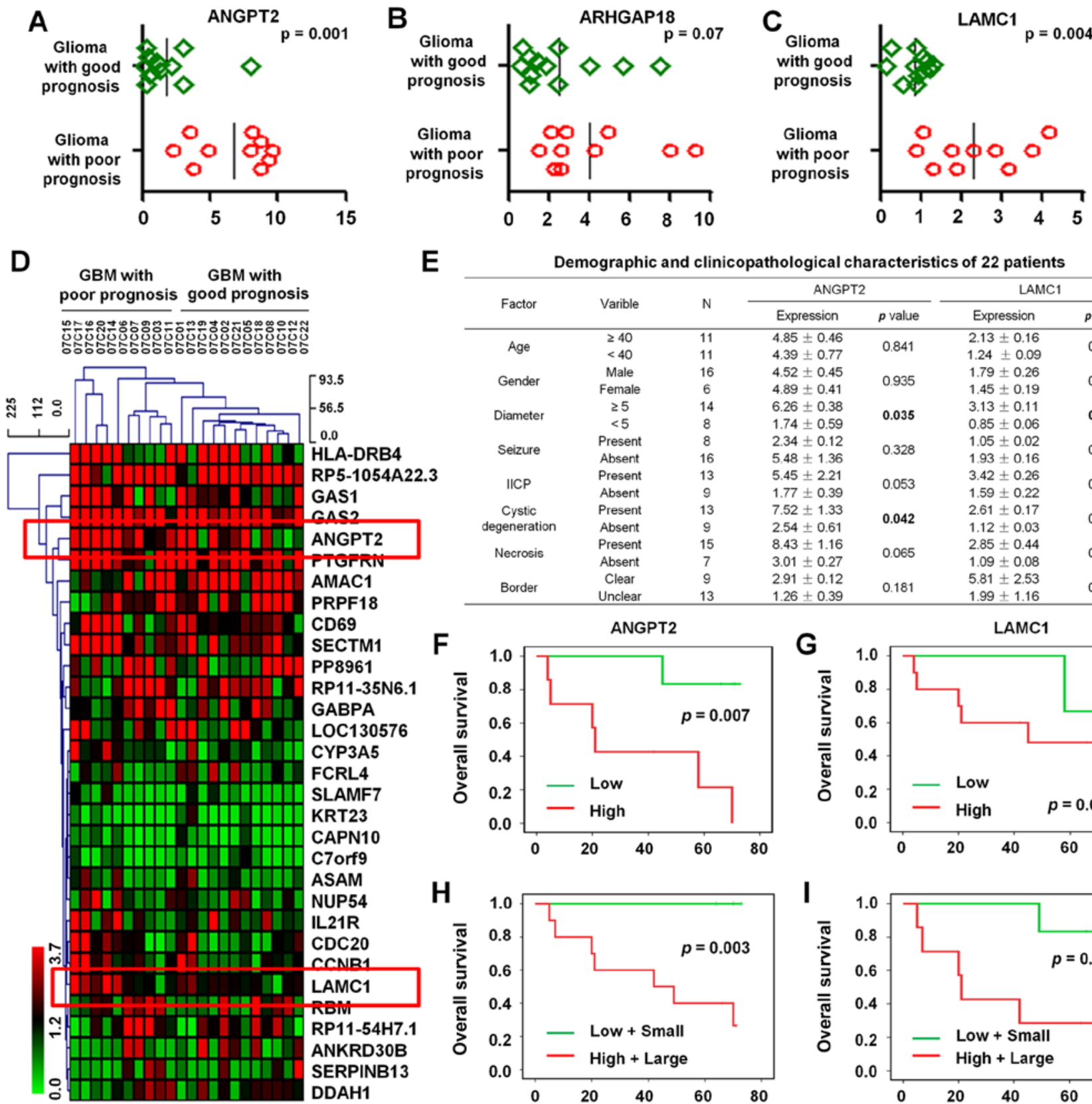

E

\begin{tabular}{|c|c|c|c|c|c|c|}
\hline \multirow{2}{*}{ Factor } & \multirow{2}{*}{ Varible } & \multirow{2}{*}{$\mathrm{N}$} & \multicolumn{2}{|c|}{ ANGPT2 } & \multicolumn{2}{|c|}{ LAMC1 } \\
\hline & & & Expression & $p$ value & Expression & $\rho$ value \\
\hline \multirow{2}{*}{ Age } & $\geq 40$ & 11 & $4.85 \pm 0.46$ & \multirow{2}{*}{0.841} & $2.13 \pm 0.16$ & \multirow{2}{*}{0.212} \\
\hline & $<40$ & 11 & $4.39 \pm 0.77$ & & $1.24 \pm 0.09$ & \\
\hline \multirow{2}{*}{ Gender } & Male & 16 & $4.52 \pm 0.45$ & \multirow{2}{*}{0.935} & $1.79 \pm 0.26$ & \multirow{2}{*}{0.656} \\
\hline & Female & 6 & $4.89 \pm 0.41$ & & $1.45 \pm 0.19$ & \\
\hline \multirow{2}{*}{ Diameter } & $\geq 5$ & 14 & $6.26 \pm 0.38$ & \multirow{2}{*}{0.035} & $3.13 \pm 0.11$ & \multirow{2}{*}{0.048} \\
\hline & $<5$ & 8 & $1.74 \pm 0.59$ & & $0.85 \pm 0.06$ & \\
\hline \multirow{2}{*}{ Seizure } & Present & 8 & $2.34 \pm 0.12$ & \multirow{2}{*}{0.328} & $1.05 \pm 0.02$ & \multirow{2}{*}{0.481} \\
\hline & Absent & 16 & $5.48 \pm 1.36$ & & $1.93 \pm 0.16$ & \\
\hline \multirow{2}{*}{$\| C P$} & Present & 13 & $5.45 \pm 2.21$ & \multirow{2}{*}{0.053} & $3.42 \pm 0.26$ & \multirow[b]{2}{*}{0.113} \\
\hline & Absent & 9 & $1.77 \pm 0.39$ & & $1.59 \pm 0.22$ & \\
\hline \multirow{2}{*}{$\begin{array}{c}\text { Cystic } \\
\text { degeneration }\end{array}$} & Present & 13 & $7.52 \pm 1.33$ & \multirow{2}{*}{0.042} & $2.61 \pm 0.17$ & \multirow[b]{2}{*}{0.097} \\
\hline & Absent & 9 & $2.54 \pm 0.61$ & & $1.12 \pm 0.03$ & \\
\hline \multirow{2}{*}{ Necrosis } & Present & 15 & $8.43 \pm 1.16$ & \multirow{2}{*}{0.065} & $2.85 \pm 0.44$ & \multirow{2}{*}{0.052} \\
\hline & Absent & 7 & $3.01 \pm 0.27$ & & $1.09 \pm 0.08$ & \\
\hline \multirow{2}{*}{ Border } & Clear & 9 & $2.91 \pm 0.12$ & \multirow{2}{*}{0.181} & $5.81 \pm 2.53$ & \multirow{2}{*}{0.069} \\
\hline & Unclear & 13 & $1.26 \pm 0.39$ & & $1.99 \pm 1.16$ & \\
\hline
\end{tabular}
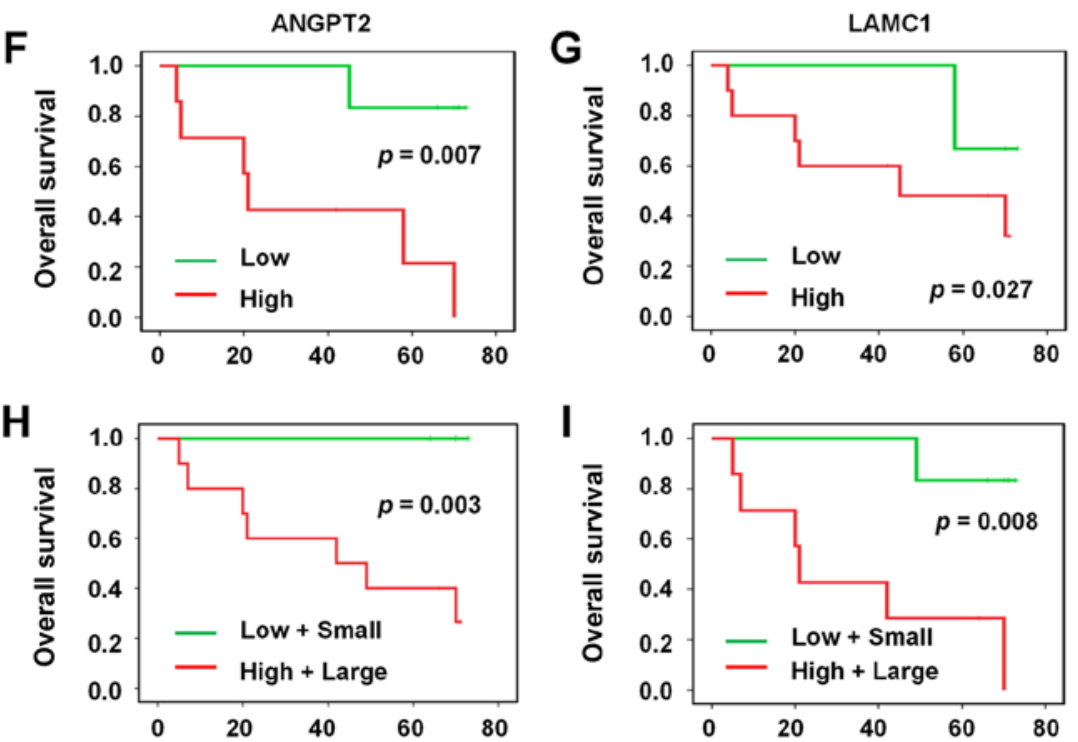

Figure 4. Clinical significance of ANGPT2 and LAMC1 in GBM. Expression level of (A) ANGPT2, (B) ARHGAP18 and (C) LAMC1 in 12 GBMs with good prognosis and $10 \mathrm{GBMs}$ with poor prognosis. (D) Hierarchical clustering for differential genes in $22 \mathrm{GBM}$ tissues. (E) Clinicopathological characteristics of 22 GBM patients. Univariate survival analysis of OS in glioma as determined by Kaplan-Meier plots estimates based on (F) ANGPT2 and (G) LAMC1 expression. Multivariate analysis of OS with a Cox proportional hazards model in GBM based on (H) ANGPT2 or (I) LAMC1 expression with tumor diameter.

when compare with normal tissues, which imply that the expression level of miR-181a was negatively correlated with ANGPT2, ARHGAP18 and LAMC1 in GBM.

Furthermore, we analyzed the correlation of miR-181a and the three genes in 42 paired GBM samples and found that the relative expression level between miR-181a and ANGPT2 presented inverse correlation $\left(\mathrm{R}^{2}=0.68\right.$; Fig. $\left.3 \mathrm{M}\right)$. In the same samples, the relative expression level between miR-181a and ARHGAP18 showed negative correlation $\left(\mathrm{R}^{2}=0.64\right.$; Fig. $\left.3 \mathrm{~N}\right)$. Similarly, the relative expression level between miR-181a and LAMC1 also indicated negative relation $\left(\mathrm{R}^{2}=0.52\right.$; Fig. $\left.3 \mathrm{O}\right)$.

Clinical significance of 3 genes in GBM. We analyzed the microarray data from 22 cases of GBM which included 12
GBM with good prognosis and 10 GBM with poor prognosis. The results showed that the expression of ANGPT2 and LAMC1 in GBM patients who had a good prognosis was significantly lower than who had a poor prognosis $(\mathrm{P}=0.001$ and 0.004 , respectively) (Fig. 4A and B). However, there is no significant difference in ARHGAP18 expression $(\mathrm{P}=0.07$; Fig. 4C). Hierarchical clustering analysis was further used to detect the expression profile of 31 differential genes (fold change $\geq 2$ or $\leq 0.5 ; \mathrm{P}<0.01$ ) in these 22 GBM patients with different prognosis, of which ANGPT2 and LAMC1 were shown to upregulate (Fig. 4D).

Next, we investigated the relationship between ANGPT2 and LAMC1 expression level and clinical characteristics. Univariate analysis of 22 cases of GBM demonstrated that 
ANGPT2 expression levels were significantly correlated with tumor diameter $(\mathrm{P}=0.035)$ and cystic degeneration $(\mathrm{P}=0.042)$ and the expression levels of LAMC1 was significantly associated with tumor diameter ( $\mathrm{P}=0.048$; Fig. 4E).

Kaplan-Meier survival analysis was then used to explore the prognostic significance of ANGPT2 and LAMC1. The results indicated high expression of ANGPT2 was negatively correlated with higher OS ( $\mathrm{P}=0.007$; Fig. 4F). Likewise, high expression of LAMC1 was negatively associated with higher OS ( $\mathrm{P}=0.027$; Fig. 4G). Therefore, high expression of ANGPT2 and LAMC1 correlated with poor prognosis in GBM patients.

Multivariate analysis of ANGPT2 expression with diameter using Kaplan-Meier estimation suggested that high expression of ANGPT2 with large diameter tumor was significantly associated with poor $\mathrm{OS}(\mathrm{P}=0.003$; Fig. $4 \mathrm{H})$. In addition, high expression of LAMC1 with large diameter tumor was also related with poor $\mathrm{OS}(\mathrm{P}=0.008$; Fig. $4 \mathrm{I})$. Take together, ANGPT2 and LAMC1 might be predictors of prognosis in GBM patients.

\section{Discussion}

GBM is one of the most deadly human malignancies worldwide and has increasing incidence with short survival rate and high mortality (15). Thus, the novel molecular mechanisms involved in the aggressive growth of GBM, and further new targeted therapies are required for prolonging the survival of glioma patients. Useful biomarkers are needed as we rely on their potential significance as a molecular targeted therapy in the treatment of GBM patients (16). Enormous efforts have been made to explore a molecular signature that would assist to cancer diagnose and therapy, in order to improve the current standard of clinical care (17). A large number of signal pathways were identified related to the development of GBM, which are meaningful characteristic biomarkers in individual patients (18).

More than 100 miRNAs were investigated that correlated with the progression of cell proliferation, metastasis and apoptosis among GBM (6). miR-181a was proven to be related to certain type of cancers (19). However, the clinical relevance and function of miR-181a in GBM remains elusive.

In the present study, via the hierarchical clustering analysis of microarray data, we found 30 upregulated and 9 downregulated miRNAs in 3 pairs of GBM, of these, miR-181a was included in the downregulated miRNAs. By systematical analysis of differential miRNAs, we discovered that miR-181a was downregulated in many cancers, especially lower expressed in GBM. Thus, miR-181a may be a tumor suppressor in GBM.

The hierarchical clustering analysis of microarray data indicated 46 upregulated and 17 downregulated genes in 3 pairs of GBM. We selected 3 upregulated genes (ANGPT2, ARHGAP18 and LAMC1), which were validated to be the target genes of miR-181a by luciferase reporter assays. Furthermore, both GO analysis and KEGG pathway analysis indicated that ANGPT2, ARHGAP18 and LAMC1 significantly associated with GBM formation. Our results showed that expression of miR-181a was negatively correlated with mRNA levels of the target genes. Moreover, high expression of ANGPT2 and LAMC1 is correlated with a shorter median OS in GBM, which suggesting that ANGPT2 and LAMC1 are underlying diagnostic biomarkers and prognostic factors for GBM.
GBM is characterized by exuberant angiogenesis, a key event in tumor growth and progression. Tumor vessel density, which represents angiogenesis, has prognostic value in various malignant tumors, including glioma (20). Angiogenesis is controlled by the interplay between numerous positive and negative factors (21). ANGPT2 is a ligand of the tyrosine kinase receptor Tie2 and integrin receptors (22). It functions as an autocrine controller of endothelial cells in a contextdependent manner, promoting either blood vessel growth or regression depending on the levels of other growth factors such as vascular endothelial growth factor (VEGF) (23). ANGPT2 level has been associated with tumor angiogenesis in various cancers (24). However, the diagnostic value of angiopoietin-2 in glioma was not investigated. Considering that the use of angiogenesis inhibitors may offer novel strategies in brain tumor therapy, our results that ANGPT2 upregulation correlated with a shorter median OS in glioma may provide new insight for diagnosis and treatment of glioma.

Tumor development and progression depend not only on the perturbed genes that govern cell proliferation and apoptosis, but is also highly determined by tumor microenvironment (TME) $(25,26)$. Extracellular matrix proteins constitute an integral part of TME and play critical roles in regulating tumor cell proliferation, survival, autophagy, migration and invasion. Although the role and molecular mechanisms in upregulation of LAMC1, an extracellular matrix glycoprotein in cancer are unclear, a previous report suggested that LAMC1 is an oncogene in breast, prostate and ovarian cancer and may contribute to the development and progression of uterine carcinoma through enhancing tumor cell motility and invasion (27), which warrants further investigation regarding its role as a biomarker and therapeutic target in cancer.

In conclusion, we have demonstrated that miR-181a is downregulated, but the target genes ANGPT2, ARHGAP18 and LAMC1 are upregulated in GBM. Our results indicated that high expression of ANGPT2 or LAMC1 in GBM is associated with shorter overall survival, which suggest that ANGPT2 and LAMC1 might be a predictor of prognosis in GBM patients.

\section{Acknowledgements}

The present study was supported in part by grants from the Key Science and Technology Project of Hainan Province (ZDXM2015070), the Social Science Development Foundation of Hainan Province (SF201414), the Natural Science Foundation of Hainan Province (813201) and the Hainan Health Institution Project (2012PT-06).

\section{References}

1. Wang H, Xu T, Jiang Y, Xu H, Yan Y, Fu D and Chen J: The challenges and the promise of molecular targeted therapy in malignant gliomas. Neoplasia 17: 239-255, 2015.

2. Demuth T and Berens ME: Molecular mechanisms of glioma cell migration and invasion. J Neurooncol 70: 217-228, 2004.

3. See SJ and Gilbert MR: Anaplastic astrocytoma: Diagnosis, prognosis, and management. Semin Oncol 31: 618-634, 2004.

4. Wang CL, Wang JY, Liu ZY, Ma XM, Wang XW, Jin H, Zhang XP, Fu D, Hou LJ and Lu YC: Ubiquitin-specific protease 2a stabilizes MDM4 and facilitates the p53-mediated intrinsic apoptotic pathway in glioblastoma. Carcinogenesis 35: 1500-1509, 2014. 
5. Wang X, Zhu Y, Ma Y, Wang J, Zhang F, Xia Q and Fu D: The role of cancer stem cells in cancer metastasis: New perspective and progress. Cancer Epidemiol 37: 60-63, 2013.

6. Srinivasan S, Patric IRP and Somasundaram K: A ten-microRNA expression signature predicts survival in glioblastoma. PLoS One 6: e17438, 2011.

7. Chen X, Hao B, Han G, Liu Y, Dai D, Li Y, Wu X, Zhou X, Yue Z, Wang L, et al: miR-372 regulates glioma cell proliferation and invasion by directly targeting PHLPP2. J Cell Biochem 116 225-232, 2015

8. Shang C, Hong Y, Guo Y, Liu YH and Xue YX: MiR-210 up-regulation inhibits proliferation and induces apoptosis in glioma cells by targeting SIN3A. Med Sci Monit 20: 2571-2577, 2014.

9. Chen W, Zhang B, Guo W, Gao L, Shi L, Li H, Lu S, Liu Y and Li X: miR-429 inhibits glioma invasion through BMK1 suppression. J Neurooncol 125: 43-54, 2015.

10. Yan Y, Zhang L, Xu T, Zhou J, Qin R, Chen C, Zou Y, Fu D, $\mathrm{Hu}$ G, Chen J, et al: SAMSN1 is highly expressed and associated with a poor survival in glioblastoma multiforme. PLoS One 8: e81905, 2013.

11. Xu T, Jiang Y, Yan Y, Wang H, Lu C, Xu H, Li W, Fu D, Lu Y and Chen J: VSIG4 is highly expressed and correlated with poor prognosis of high-grade glioma patients. Am J Transl Res 7: $1172-1180,2015$.

12. Qin R, Zhou J, Chen C, Xu T, Yan Y, Ma Y, Zheng Z, Shen Y, $\mathrm{Lu} \mathrm{Y,Fu} \mathrm{D,} \mathrm{et} \mathrm{al:} \mathrm{LIN28} \mathrm{is} \mathrm{involved} \mathrm{in} \mathrm{glioma} \mathrm{carcinogenesis} \mathrm{and}$ predicts outcomes of glioblastoma multiforme patients. PLoS One 9: e86446, 2014.

13. Wang Q, Wang JY, Zhang XP, Lv ZW, Fu D, Lu YC, Hu GH, Luo C and Chen JX: RLIP76 is overexpressed in human glioblastomas and is required for proliferation, tumorigenesis and suppression of apoptosis. Carcinogenesis 34: 916-926, 2013.

14. Vlachos IS, Paraskevopoulou MD, Karagkouni D, Georgakilas G, Vergoulis T, Kanellos I, Anastasopoulos IL, Maniou S, Karathanou K, Kalfakakou D, et al: DIANA-TarBase v7.0: indexing more than half a million experimentally supported miRNA:mRNA interactions. Nucleic Acids Res 43 (D1): D153-D159, 2015.

15. Manterola L, Guruceaga E, Gállego Pérez-Larraya J, GonzálezHuarriz M, Jauregui P, Tejada S, Diez-Valle R, Segura V, Samprón N, Barrena C, et al: A small noncoding RNA signature found in exosomes of GBM patient serum as a diagnostic tool. Neuro Oncol 16: 520-527, 2014.
16. Tumilson CA, Lea RW, Alder JE and Shaw L: Circulating microRNA biomarkers for glioma and predicting response to therapy. Mol Neurobiol 50: 545-558, 2014.

17. Zemp FJ, McKenzie BA, Lun X, Reilly KM, McFadden G, Yong VW and Forsyth PA: Cellular factors promoting resistance to effective treatment of glioma with oncolytic myxoma virus. Cancer Res 74: 7260-7273, 2014

18. Grant R, Kolb L and Moliterno J: Molecular and genetic pathways in gliomas: The future of personalized therapeutics. CNS Oncol 3: 123-136, 2014.

19. Shi L, Cheng Z, Zhang J, Li R, Zhao P, Fu Z and You Y: hsa-miR181a and hsa-mir-181b function as tumor suppressors in human glioma cells. Brain Res 1236: 185-193, 2008.

20. Wu SD, Ma YS, Fang Y, Liu LL, Fu D and Shen XZ: Role of the microenvironment in hepatocellular carcinoma development and progression. Cancer Treat Rev 38: 218-225, 2012.

21. Wang Y, Ma Y, Fang Y, Wu S, Liu L, Fu D and Shen X: Regulatory T cell: A protection for tumour cells. J Cell Mol Med 16: 425-436, 2012

22. Janelidze S, Lindqvist D, Francardo V, Hall S, Zetterberg H, Blennow K, Adler CH, Beach TG, Serrano GE, van Westen D, et al: Increased CSF biomarkers of angiogenesis in Parkinson disease. Neurology 85: 1834-1842, 2015.

23. Holash J, Wiegand SJ and Yancopoulos GD: New model of tumor angiogenesis: Dynamic balance between vessel regression and growth mediated by angiopoietins and VEGF. Oncogene 18: 5356-5362, 1999.

24. Tsai YC, Lee CS, Chiu YW, Kuo HT, Lee SC, Hwang SJ, Kuo MC and Chen HC: Angiopoietin-2 as a prognostic biomarker of major adverse cardiovascular events and all-cause mortality in chronic kidney disease. PLoS One 10: e0135181, 2015.

25. Stewart TA, Yapa KT and Monteith GR: Altered calcium signaling in cancer cells. Biochim Biophys Acta 1848 (10 Pt B): 2502-2511, 2015

26. Han DY, Fu D, Xi H, Li QY, Feng LJ, Zhang W, Ji G, Xiao JC and Wei Q: Genomic expression profiling and bioinformatics analysis of pancreatic cancer. Mol Med Rep 12: 4133-4140, 2015.

27. Kashima H, Wu RC, Wang Y, Sinno AK, Miyamoto T, Shiozawa T, Wang TL, Fader AN and Shih IeM: Laminin C1 expression by uterine carcinoma cells is associated with tumor progression. Gynecol Oncol 139: 338-344, 2015. 